\title{
BMJ Open Ageing and mental health: changes in self-reported health due to physical illness and mental health status with consecutive cross-sectional analyses
}

\author{
Geir Fagerjord Lorem, ${ }^{1}$ Henrik Schirmer, ${ }^{2,3}$ Catharina E A Wang, ${ }^{4,5}$ Nina Emaus ${ }^{1}$
}

To cite: Lorem GF, Schirmer $\mathrm{H}$, Wang CEA, et al. Ageing and mental health: changes in self-reported health due to physical illness and mental health status with consecutive cross-sectional analyses. BMJ Open 2017;7: e013629. doi:10.1136/ bmjopen-2016-013629

- Prepublication history and additional material is available. To view please visit the journal (http://dx.doi.org/ 10.1136/bmjopen-2016013629).

Received 27 July 2016 Revised 2 December 2016 Accepted 6 December 2016

CrossMark

For numbered affiliations see end of article.

Correspondence to Professor Geir Fagerjord Lorem; geir.lorem@uit.no

\section{ABSTRACT}

Objectives: It is known that self-reported health (SRH) declines with increasing age and that comorbidity increases with age. We wished to examine how age transfers its effect to SRH through comorbid disease and mental illness and whether these processes remained stable from 1994 until 2008. The hypothesis is that ageing and/or the increased age-related burden of pathology explains the declining $\mathrm{SRH}$.

Setting: The Tromsø Study (TS) is a cohort study using a survey approach with repeated physical examinations. It was conducted in the municipality of Tromsø, Norway, from 1974 to 2008.

Participants: A total of 21199 women and 19229 men participated.

Primary and secondary outcome measures: SRH is the outcome of interest. We calculated and compared the effect sizes of age, comorbidity and mental health symptoms using multimediator analysis based on OLS regression.

Results: Ageing had a negative impact on $\mathrm{SRH}$, but the total effect of age decreased from 1994 to 2007. We assessed the direct effect of age and then the proportion of indirect age-related effects through physical illness and mental health symptoms on the total effect. The direct effect of age represented $79.3 \%$ of the total effect in 1994 and decreased to $58.8 \%$ in 2007. Physical illness emerged as an increasingly important factor and increased its influence from $15.7 \%$ to $41.2 \%$ of the total effect. Age alone had a protective effect on mental health symptoms and this increased (2.5\% to $17.3 \%)$, but we found a stronger association between mental health symptoms and physical disease in the later waves of the study (increasing from $3.7 \%$ to $14.8 \%$ ).

Conclusions: The results suggest that the effect on $\mathrm{SRH}$ of mental health symptoms caused by physical illness is an increasing public health problem. Treatment and care for specific medical conditions must therefore focus more strongly on how these conditions affect the patient's mental health and address these concerns accordingly.

\section{INTRODUCTION}

Self-reported health ( $\mathrm{SRH}$ ) is a subjective assessment of current health status as seen by

\section{Strengths and limitations of this study}

- The sample comprises large, representative samples of a general population with repeated measures at $\sim 7$-year intervals.

- Multimediator analysis allows for the interpretation of the joint effect of age, comorbid disease and mental health on self-reported health.

- We used the repeated measures as separate cross-sectional data in the analysis.

- The first three panels (1974-1986) did not include any adequate measurement of mental health symptoms and were excluded, but the CONOR-MHI (1994) was validated against the Hopkins Symptom Checklist and showed good agreement.

the patient or participant. It is well known that a whole range of biological, psychological and socioeconomic factors affect $\mathrm{SRH}$, and also that these factors interact. ${ }^{1-5}$ The research literature suggests that $\mathrm{SRH}$ is produced in a cognitive process that is inherently subjective and contextual, and also that SRH predicts mortality and other health outcomes; this shows that the basis of self-rated health lies in the biological and physiological state of the individual organism. ${ }^{6}$ Well-known crucial biological factors that independently affect SRH are specific medical conditions (eg, cardiovascular diseases, diabetes and asthma) and health risk factors (eg, resting heart rate, blood pressure, cholesterol, BMI and endocrine measures). Although the effect of SRH attenuates when such variables are controlled for, SRH still remains as an independent variable for all-cause death and other future health outcomes. ${ }^{7-11}$ Mental health symptoms affect SRH, but mental health is also affected by physical disease. The literature suggests that severity of mental health symptoms correlates with many specific medical conditions, and consequently with impaired well-being. Comorbid strain increases with increasing age, and older 
people are particularly at risk of experiencing anxiety and depression. ${ }^{12-17}$

To summarise, it is well documented that SRH declines with increasing age but whether it is ageing alone or the increased age-related burden of pathology that explains this association is still unanswered. The prevalence of coexisting chronic conditions is rising as life expectancy increases in contemporary Western society. ${ }^{18}$ The age-specific decline could mean that the increasing level of pathology due to age explains this specific decline of SRH and not ageing by itself.

There are, to the best of our knowledge, no studies that describe the combined effect of ageing, comorbid physical disease and mental health symptoms on general perceived health status. Moreover, since medical treatment has improved over the past three decades, leading to increased life expectancy, it seems timely to ask whether people's experiences of ageing, comorbid disease and mental health problems remain the same. We wished to examine how age transfers its effect on SRH through comorbid physical disease and mental health symptoms. A further aim was to explore how mental health symptoms are affected by physical disease and whether these processes remained stable from 1986 until 2008.

\section{METHOD}

\section{Sample and design}

The Tromsø Study (TS) was a cohort study which provided data allowing us to estimate the impact of a broad range of factors on a general population, using surveys and physical examinations in a large representative sample. ${ }^{19}$ TS consisted of six surveys conducted in Tromsø in Northern Norway from 1974 to 2008. We used consecutive cross-sectional analyses within the TS. The study population was recruited from all inhabitants in specific age groups. The aim was to include large, representative samples of the Troms $\varnothing$ population, with the invitation of whole birth cohorts and random samples. The attendance rate was high (66-75\%). A total of 21199 women and 19229 men gave informed signed consent and attended up to 6 separate health examinations. Troms $\varnothing 1$ was a heart study conducted in 1974 and included only men aged 20-49. Troms $\varnothing 2$ followed-up the first study in 1979-1980 but included men (aged 20-54) and women (aged 20-49). Tromsø 3 was executed in 1986-1987 and included men and women in the 20-56 age range, and a $10 \%$ random selection of persons aged 12-19. We excluded Troms $\varnothing$ 1-3. SRH was introduced during the 1980s; Tromsø 1 and 2 thus lack SRH and Tromsø 3 did not include any adequate measurement of mental health symptoms. Our sample starts with Troms $\varnothing 4$ in 1994. Troms $\varnothing 4$ is the largest wave, and participants were followed-up in 2001 and $2007 / 2008$. We excluded those with missing data $(\mathrm{n}=736$ in TS4, $\mathrm{n}=1132$ in TS5 and $\mathrm{n}=767$ in TS6). The final analysis therefore comprised 12408 men and
13579 women from TS4, 3108 men and 3746 women from TS5 and 5769 men and 6338 women from TS6. The Norwegian Data Protection Authority and the Regional Committees for Medical and Health Research Ethics North Norway approved the TS.

\section{Measurements}

The participants completed a self-administrated questionnaire with questions on a broad range of diseases and symptoms, health behaviour, social conditions, education, financial situation and level of physical activity. Self-reported health (SRH): The independent variable SRH was reported by answering the question, 'What is your current state of health?' with answers ranging from very bad (0) to very good (4) in Tromsø 6, and from poor (1) to very good (4) in Tromsø 4 and 5. Specific medical conditions: We selected 13 symptomatic medical conditions reported in all panels. These were psoriasis, food allergies, chronic bronchitis, migraine, ulcer, asthma, thyroid disease, arthritis, myocardial infarction, cerebrovascular stroke, diabetes, osteoporosis and angina. The conditions were self-reported by answering questions such as 'Do you have or have you had....?' We used the Health Impact Index (HII) to measure the comorbid conditions. Diseases have a varied impact on SRH. HII classifies patients with comorbid disease according to the impact that each condition has on SRH by assigning a weight for each condition. HII equals the total score of each condition of the participant. HII thus considers the severity and joint effects of the conditions. ${ }^{4}$ The range was $0-18$ in TS4, $0-17$ in TS5 and 0-22 in TS 6. Online supplementary appendix 1 shows the conditions included with their weights and prevalence in the different waves.

Mental health symptoms were based on a wellvalidated self-report symptom inventory comprising questions representative of the symptom configurations of anxiety and depression commonly observed among outpatients. It includes questions such as 'Have you experienced sudden fear without apparent reason', '... felt tense or upset', '...easily blamed yourself', '...felt depressed or sad', '...felt useless or worthless', '...felt that everything is a struggle' or '...felt hopelessness'. Each answer is scored from 1 to 4 . The measurement is the average score. The range was therefore 1-4 in all waves. The mental health index (CONOR-MHI) used at T4 has been compared with the Hopkins Symptom Checklist (HSCL) with reasonably good agreement. In the following surveys T5-6, the HSCL was used. A cut-off level of 2.15 for significant symptoms is equivalent to the 1.85 level in HSCL-10. ${ }^{20} 21$

\section{Analysis}

The purpose of the descriptive statistics was to define the distribution of SRH, comorbid disease and mental health across samples, age groups and gender. We used cross tabulation and two-way ANOVA to describe the characteristics of the sample. Multimediator analysis was 
used for the analysis of the conditional nature of the mechanism by which age transmits its effect on SRH. The advantage of this method is that it allows for the interpretation of multiple confounders that may function as either mediators or moderators and interprets their joint effect on the statistical model derived from the theoretical model. ${ }^{22} 23$ The analytical goal of the multimediation analysis was to determine how age transfers its effect to SRH directly and through physical disease and mental illness. The first step was the conceptual model, which we based on the idea that age represents the timeline of life in which events like disease occur and physical condition changes. Previous analysis, tracking individual subjects, confirms that SRH decreases with increasing age and whenever levels of pathology increase. This implies that age might influence SRH either directly or indirectly through pathology as life events. The second step was to translate the conceptual model into a statistical model. Figure 1 shows the conceptual model and its translation into a statistical model. ${ }^{24}$ The statistical model includes SRH as the outcome $(\mathrm{Y})$, age as the main variable $(\mathrm{X})$ with medical condition $\left(\mathrm{M}_{1}\right)$ and mental health symptoms $\left(\mathrm{M}_{2}\right)$ as mediators. Our statistical model includes three indirect effect lines (Ind 1-3).

- Ind 1: Age $\rightarrow \mathrm{HII} \rightarrow \mathrm{SRH}\left(\mathrm{a}_{1} \times \mathrm{b}_{1}\right)$

- Ind 2: Age $\rightarrow \mathrm{HII} \rightarrow \mathrm{HSCL} \rightarrow \mathrm{SRH}\left(\mathrm{a}_{1} \times \mathrm{d}_{21} \times \mathrm{b}_{2}\right)$

- Ind 3: Age $\rightarrow \mathrm{HSCL} \rightarrow \mathrm{SRH}\left(\mathrm{a}_{2} \times \mathrm{b}_{2}\right)$

We used multiple regression to assess the two mediators $\left(\mathrm{M}_{1}=\right.$ medical conditions and $\mathrm{M}_{2}=$ mental health $)$ and the reaction $(\mathrm{Y}=\mathrm{SRH})$. The regression coefficients, 95\% CIs and model summary information for the mediated effect of age on SRH have been published as online supplementary appendix 2 .

\section{RESULTS}

\section{Characteristics and total effect of age}

Table 1 shows the characteristics of the four samples indicating increasing comorbidity with a shift in 2001 (T5) when the comorbid levels decreased with a corresponding increase in SRH. Figure 2 shows profile plots for SRH, comorbidity and ratio of persons with subthreshold and significant mental health symptoms across age and gender. Testing for gender and age differences with two-way ANOVA showed that all mean differences were significant $(p<0.0001)$ for SRH. Here, SRH declined significantly with increasing age with a corresponding increase in comorbidity at all three survey points. Although the gender differences were statistically significant for all three factors, the gender difference in SRH was less than a 10-year age difference in SRH in all waves. For comorbidity, the gender difference was as large as a 10-year age difference for the two intermediate survey points, but less so at the first and last survey points where the gender difference was small. For comorbidity, the most striking finding was the increase

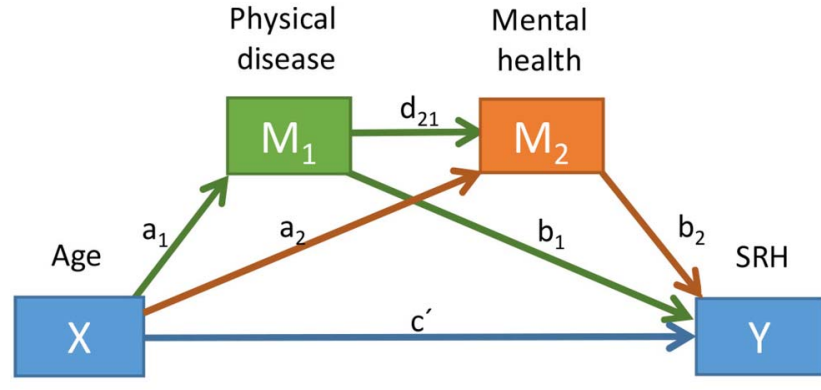

Indirect effect of $X$ on $Y$ through $M_{i}=a_{i}{ }^{*} b_{i}$

Indirekt effect of $X$ on $Y$ thorugh $M_{1}$ and $M_{2}$ in serial $=a_{1}{ }^{*} d_{21}{ }^{*} b_{2}$ Direct effect of $X$ on $Y=c^{\prime}$

Figure 1 Conceptual and statistical diagram for the mediated effect of age on SRH through comorbid disease and mental health symptoms.

Table 1 Distribution of SRH, physical condition and demographics, specific medical conditions, mental health symptoms and social context by gender in Troms $ø$ 4-6 (1994-2008)

Tromsø 4 Tromsø 5 Tromsø 6

\begin{tabular}{lll}
\hline Self-rated health & $2.82(0.70)$ & $2.7(0.67) 2.74(0.77)$
\end{tabular}

(mean/SD)

Age (mean/SD)

$48.1(14.8) 60.1(13.8) 58.7(12.4)$

Health impact index

(mean/SD)

Mental health

$0.95(1.66) 1.72(2.18) 1.66(2.21)$

symptoms (mean/SD)

$\mathrm{SRH}$, self-reported health.

by age across all surveys, especially for women, who had an increasing burden of disease as they got older. For mental health symptoms, the greater burden for women was most striking.

\section{The total effect of age}

We used an OLS regression model to determine the total effect of age on SRH. Table 2 shows the linear model of the total effect of age on SRH. We see that age had a negative effect on SRH in all samples, and also that the total effect of age attenuated from 1994 to 2008 in parallel with increasing life expectancy in the region. In our model, each year of age represented -0.0175 (CI -0.018 to -0.017$)$ deterioration of SRH in T4 but the effect dropped to -0.013 (CI -0.014 to -0.012 ) in T6.

\section{The indirect effect of pathology}

The $\mathrm{M}_{1}$ models in online supplementary appendix 2 show that higher comorbidity was associated with increasing age in all waves (Coeff $=0.050$ in T4; 0.059 in $\mathrm{T} 5 ; 0.050$ in $\mathrm{T} 6$ ). The $\mathrm{M}_{2}$ models show a significant effect for age on mental health symptoms (Coeff= -0.0002 in $\mathrm{T} 4 ;-0.0025$ in $\mathrm{T} 5 ;-0.0029$ in T6), although 


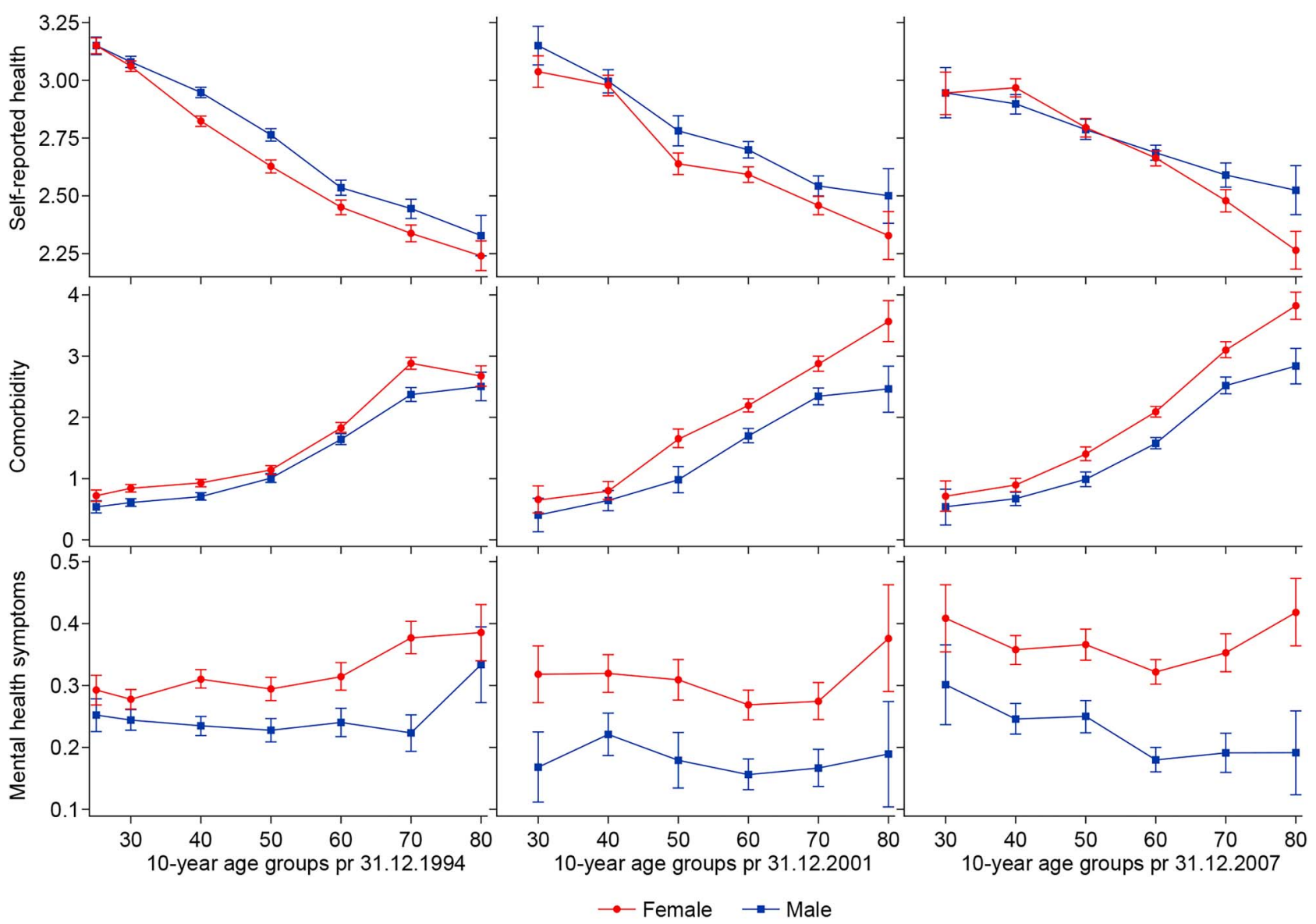

Figure 2 Profile plots for self-reported health for interaction effects between age and gender with $95 \%$ Cls. Self-reported health: Range from very poor (0) to very good (4) in TS 6, and poor (1) to very good (4) in TS 4 and 5. Comorbid disease: Number of diseases grouped into a score with range 0-17 (mean 0.97) in TS 4, range 0-17 (mean 1.59) in TS 5 and range 0-19 (mean 1.53) TS 6. Mental health symptoms: CONOR-MHI with range 1-4 (mean 1.52) in TS 4, and HSCL-10 with range 1-4 in TS 5 and 6 (mean 1.25 in TS 5 and mean 1.29 in TS 6). All differences $p<0.001$. Red lines=women, blue lines=men, $\mathrm{Cl} 95 \%$ is $\mathrm{SE}^{\star} 1.96$.

medical conditions when they occurred affected mental health symptoms more than age $(0.030$ in T4; 0.032 in $\mathrm{T} 5 ; 0.041$ in T6). All effect lines in the statistical model were estimated by a series of OLS regression models (see online supplementary appendix 2). Table 3 shows the indirect and direct effects of age on SHR. We calculated these from the coefficients in online supplementary appendix 2 according to our statistical model. Adding gender as a moderator on each effect line did not change the overall results.

We found that age had a direct and indirect effect on SRH. The direct effect $\left(c^{\prime}\right)$ of age attenuated from 1994 to 2008 (T4: $c^{\prime}=-0.013$, T5: $\left.c^{\prime}=-0.011, T 6: c^{\prime}=-0.008\right)$. This suggests that age affected SRH independently of pathology even when controlling for the mediators, and also that age itself had a lower impact on SRH at the latest measuring point.

We found that age had an increasing negative indirect effect through comorbid diseases (T4: -0.0034 ; T5: -0.0035 ; T6: -0.0042$)$. Since the total effect attenuated in the same period, this implied that the ratio of total to indirect effect of comorbid disease increased correspondingly more. It was 0.192 in $1994,0.236$ in 2001 and 0.330 in $2007 / 2008$. This trend implied that physical disease was an increasingly important factor relative to age itself to explain why SRH declines with increasing age.

The second indirect effect (Age $\rightarrow \mathrm{HII} \rightarrow \mathrm{HSCL} \rightarrow \mathrm{SRH}$ ) included mental health symptoms associated with having a disease. We found a negative effect on SRH T4 of -0.0006 , T5 of -0.0013 and $\mathrm{T} 6$ of -0.0019 . This suggests that having a physical disease was associated with higher levels of mental health symptoms, which in turn affected SRH. The ratio of total to indirect effect of comorbid disease was -0.037 in $\mathrm{T} 4,-0.086$ in $\mathrm{T} 5$ and -0.148 in T6. Thus, we see a consistent increase in the relative size of the second indirect effect from 1994 to 2007/2008. This implied that the relative significance of mental health issues connected to physical disease increased during this period and at $14.8 \%$ of the total effect, it is also clinically significant.

The third indirect effect line (Age $\rightarrow$ HSCL $\rightarrow$ SRH) revealed that SRH increased with increasing age, which implies that mental health symptoms are associated with 


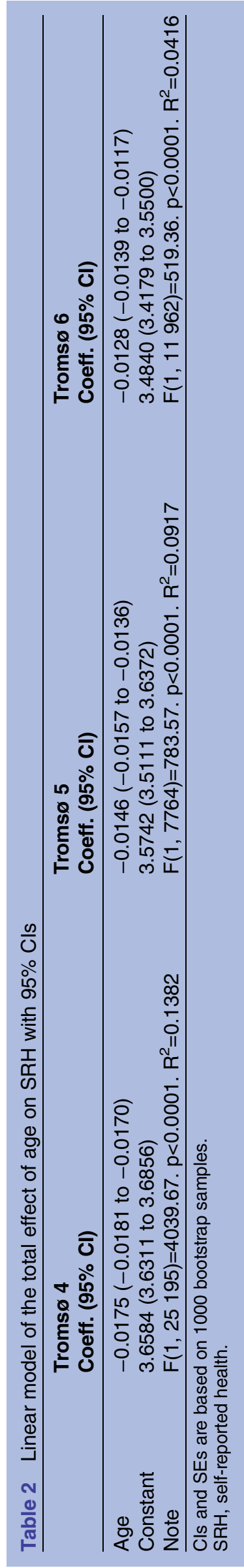

increasing age when controlled for physical disease. The ratio of effect size increased during this period from 0.002 in $\mathrm{T} 4$ to -0.0109 in $\mathrm{T} 5$ and -0.200 in T6. This implied that when we disregard physical illness and mental health problems associated with physical illness, increasing age had a beneficial effect on SRH.

\section{DISCUSSION}

Ageing affected SRH directly and also indirectly through increased levels of pathology. We observed a change in how ageing and physical disease influenced SRH between the different waves. The direct effect of ageing $\left(\mathrm{c}^{\prime}\right)$ represented $79.3 \%$ of the total effect in $1994,69.8 \%$ in 2001 and $58.8 \%$ in $2007 / 2008$. This means that ageing is still the most important factor for SRH, but that ageing itself became relatively less important between the waves. Meanwhile, physical disease became an increasingly important factor for SRH. As shown in table 3, comorbid conditions (HII) represented $15.7 \%$ of the total effect in $1994,26.7 \%$ in 2001 and $41.2 \%$ in the last wave in $2007 / 2008$. Furthermore, ageing itself had a protective effect on mental health symptoms which increased (2.0-20.0\% of the total effect). We found a stronger association between mental health symptoms and physical disease in the later waves (increasing from $3.7 \%$ to $14.8 \%$ ). Mental health symptoms related to physical disease consequently led to lower SRH levels in the later parts of the study.

Physical disease is known to be related to mental health symptoms of anxiety and depression, which the HSCL-10 scale is especially sensitive to measure in a general population. ${ }^{15}$ Epidemiological data suggest that severity of mental health symptoms correlates with disease, for example, one-third of stroke survivors develop depression ${ }^{12}$ and one-quarter anxiety disorders. ${ }^{13}$ Cardiovascular diseases have shown discrete effects for panic disorder and specific phobia. ${ }^{14}{ }^{15}$ Older people with illnesses such as coronary heart disease, arthritis and chronic lung disease show increased levels of depressed mood and impaired well-being. ${ }^{16}$ Cumulative stress exposure across different stress domains contributes to depressive symptoms in cancer survivors. ${ }^{17}$ Moreover, pessimism, negative cancer-related rumination and physical symptom distress predicted anxiety and depression trajectories.

However, our findings indicate that physical disease in recent decades has become more strongly associated with mental health symptoms, that is, the indirect effect on mental health symptoms via physical disease has increased over time. Accordingly, it seems plausible that physical diseases in terms of SRH affect us more than before, and also that physical disease has a greater impact on our reaction towards illness than before. So how can we explain these findings? Why does physical disease trigger symptoms of anxiety and depression more often than before? 
Table 3 Direct and indirect effect size with 95\% bias corrected Cls in parentheses, SEs and ratio of indirect to direct effect of age on self-reported health

\begin{tabular}{|c|c|c|c|}
\hline & $\begin{array}{l}\text { Tromsø } 4 \\
\text { Effect }(95 \% \mathrm{Cl})\end{array}$ & $\begin{array}{l}\text { Tromsø } 5 \\
\text { Effect }(95 \% \mathrm{Cl})\end{array}$ & $\begin{array}{l}\text { Tromsø } 6 \\
\text { Effect }(95 \% \mathrm{Cl})\end{array}$ \\
\hline \multicolumn{4}{|c|}{ Indirect effect of age on $\mathrm{SRH}$} \\
\hline Total & $-0.0034(-0.0032$ to -0.0037$)$ & $-0.0039(-0.0034$ to -0.0043$)$ & $-0.0046(-0.0040$ to -0.0052$)$ \\
\hline $\mathrm{Age} \rightarrow \mathrm{HII} \rightarrow \mathrm{SRH}$ & $-0.0027(-0.0028$ to -0.0027$)$ & $-0.0043(-0.0044$ to -0.0041$)$ & $-0.0053(-0.0054$ to -0.0051$)$ \\
\hline $\mathrm{Age} \rightarrow \mathrm{HII} \rightarrow \mathrm{HSCL} \rightarrow \mathrm{SRH}$ & $-0.0006(-0.0006$ to -0.0007$)$ & $-0.0013(-0.0011$ to -0.0014$)$ & $-0.0019(-0.0017$ to -0.0021$)$ \\
\hline Age $\rightarrow \mathrm{HSCL} \rightarrow \mathrm{SRH}$ & $0.000 *(0.0002$ to -0.0002$)$ & $0.0016(0.0021$ to 0.0011$)$ & $0.0026(0.0032$ to 0.0020$)$ \\
\hline \multicolumn{4}{|c|}{ Ratio of indirect to total effect of age on $\mathrm{SRH}$} \\
\hline Total & $0.195(0.176$ to 0.215$)$ & $0.267(0.217$ to 0.318$)$ & $0.360(0.284$ to 0.442$)$ \\
\hline Age $\rightarrow \mathrm{HII} \rightarrow \mathrm{SRH}$ & $0.157(0.154$ to 0.158$)$ & $0.290(0.281$ to 0.299$)$ & $0.412(0.391$ to 0.433$)$ \\
\hline $\mathrm{Age} \rightarrow \mathrm{HII} \rightarrow \mathrm{HSCL} \rightarrow \mathrm{SRH}$ & $0.037(0.031$ to 0.043$)$ & $0.086(0.071$ to 0.102$)$ & $0.148(0.122$ to 0.179$)$ \\
\hline $\mathrm{Age} \rightarrow \mathrm{HSCL} \rightarrow \mathrm{SRH}$ & $0.002^{*}(-0.010$ to 0.013$)$ & $-0.109(-0.135$ to -0.083$)$ & $-0.200(-0.229$ to -0.170$)$ \\
\hline
\end{tabular}

One possible explanation may be found in social changes in Norway and the Norwegian healthcare system. Although we today have curative and palliative treatment of many more physical disorders, and more individuals have access to treatment, there is also an increased expectation of 'active ageing' and healthy living. ${ }^{25}{ }^{26}$ This expectation is realistic, as the incidence of especially cardiovascular diseases has been rapidly declining for several decades, but is contrasted by a decreasing case fatality, leaving more of those who still get cancer, coronary heart attacks and stroke with lasting disability as more people survive. ${ }^{27}$

SRH can reflect the states of the human body and mind. People base their health assessments on different types of information and contextual frameworks. ${ }^{6}$ It is plausible that people who expect to age actively become unhappy or worried when encountering limitations and disease. It may also be argued that people tend to respond negatively to questions on their health or limitations when comparing their situation with others at similar age. Bodily sensations that are directly available to the individual are another source of information. ${ }^{6}$ Accordingly, it seems plausible that people compare current body status with the situation before the disease occurred, and experience fear of relapse or having another disease. We cannot answer this assumption based on three cross-sectional analyses; however, it is a hypothesis that could be answered by tracking individuals in the TS cohort.

Furthermore, current healthcare services are organised to place greater emphasis on efficiency than on care, and society has a faster pace of life so that older people more often live alone and isolated than a few decades ago. From an evolutionary perspective, symptoms of anxiety and depression are understood as normal reactions to life-threatening and uncontrollable situations. For example, fear is an obvious adaptive function as it stimulates the 'fight-or-flight' response when the individual is exposed to a threat or dangerous situation; unless the individual can escape, it will hide or 'freeze' the situation. ${ }^{28}$ Furthermore, Gilbert describes anxiety disorders as a maladaptive expression or phenotype of the original functional fear system where the acute stress response is triggered in an inappropriate manner. ${ }^{29}$ Similarly, Nettle proposes that depression may represent a maladaptive expression of an original functional control system for positive affect, that is, a functional downregulation of positive affect in certain situations and contexts. ${ }^{30}$ Gilbert describes such a downregulation of positive affect as a defensive reaction, a similar fight-or-flight response, in situations where the individual experiences loss of control over aversive events or over significant resources including the social environment. ${ }^{29}$ An increased incidence of comorbid physical disorders with consequent reduced access to social participation can thus be a plausible explanation of an increase in mental symptoms related to physical disorders.

Interestingly, we found that age by itself was protective of mental health symptoms when controlled for the mental health symptoms associated with physical illness. Several studies focus on how physical disease is associated with increased risk of mental health symptoms. In our study, this mechanism represented $5 \%$ of the total effect in 1994, 6\% in 2001 and $12 \%$ in 2008. Our findings concur with studies on patient populations showing that mental health is an important aspect of impairment of SRH when physical illness occurs.

\section{Strengths and limitations of the method}

HII includes 13 symptomatic medical conditions, but does not include risk factors such as hypertension and dyslipidemia. These could be included as mediators on the age- $>$ HII- $>$ SRH effect line, but this did not change the overall findings of the model. The TS includes 
cancer but it is self-reported and does not distinguish between those with an active illness and those who have had cancer. That was the most likely explanation for why it did not add to the model ${ }^{4}$ and was therefore not included.

Although measured on an ordinal scale, the underlying phenomenon of SRH is continuous, and the scales represent similar logical increments. Furthermore, the distribution of SRH, apart from being staggered, resembled the shape of a normal distribution. Hence, an OLS regression model could be used for the analysis of independent associations in the multivariable model. ${ }^{22}$ Adding gender as a moderator on each effect line did not change the overall results. Mental health symptoms were measured with different instruments, which may have affected our findings. T5 and T6 used the Hopkins Symptom Checklist (HSCL-10), which is a self-reported symptom inventory comprising 10 items representative of the symptom configurations of anxiety and depression commonly observed among outpatients. ${ }^{20} \mathrm{~T} 4$ used the CONOR Mental Health Index (MHI). This was based on seven questions concerning different symptom configurations of anxiety and depression. It was partly derived from HSCL-10 and the General Health Questionnaire (GHQ). Fortunately, Troms $\varnothing 4$ is included in the CONOR database that also included HSCL-10. The index has therefore been compared with HSCL-10 with reasonably good agreement. It has been concluded that the scales can be used in epidemiological studies. For comparisons, it is recommended to use the cut-off level of 2.15 for significant symptoms as equivalent to the 1.85 level in HSCL-10. ${ }^{21} 31$

\section{CONCLUSIONS}

As medicine advances and life expectancy increases, we have higher expectations for the healthcare system and to remain healthy even in old age. The results suggest that the effect on SRH of mental health symptoms caused by physical illness is an increasing public health problem. It seems that our resilience to diseases is decreasing. Therefore, treatment and care for specific medical conditions must focus more strongly on how these conditions affect the patient's mental health and address these concerns accordingly.

\section{Author affiliations \\ ${ }^{1}$ Department of Health and Care Sciences, Faculty of Health Sciences, The Arctic University of Norway, Tromsø, Norway \\ ${ }^{2}$ Department of Clinical Medicine, Faculty of Health Sciences, The Arctic University of Norway, Tromsø, Norway \\ ${ }^{3}$ Division of Cardiothoracic and Respiratory Medicine, University Hospital of Northern Norway, Tromsø, Norway \\ ${ }^{4}$ Department of Psychology, Faculty of Health Sciences, The Arctic University of Norway, Tromsø, Norway \\ ${ }^{5}$ Division of Child and Adolescent Health, University Hospital of Northern Norway, Tromsø, Norway}

Acknowledgements The authors thank all participants in the Tromsø study and all members of the Tromsø study team. They also thank Tom Wilsgaard for his statistical advice.
Contributors GFL, HS, CEAW and NE had full access to all of the data in the study and take responsibility for the integrity of the data and the accuracy of the data analysis; involved in acquisition, analysis or interpretation of data and contributed to critical revision of the manuscript for important intellectual content. GFL, HS and NE involved in study concept and design. GFL contributed to statistical analysis and drafting of the manuscript. The Tromsø Study of UiT The Arctic University of Norway provided the data.

Funding This study was supported by UiT The Arctic University of Tromsø (EUTRO 8010.00055).

Competing interests None declared.

Patient consent Obtained.

Provenance and peer review Not commissioned; externally peer reviewed.

Data sharing statement We received the data from the Tromsø study. The data contain sensitive health information about the participants. Data cannot be made publicly available without compromising participant confidentiality and privacy. Directives from the Research Ethical Committee and the Norwegian Data Protection Authority thus prohibit us from making the minimal data set publicly available. Data are available from the Tromsø study for researchers who meet the criteria for access to confidential data (https:// en.uit.no/prosjekter/prosjekt?p_document_id=80172). Furthermore, all variables are described in the NESSTAR database: http://tromsoundersokelsen. uit.no/webview/.

Open Access This is an Open Access article distributed in accordance with the Creative Commons Attribution Non Commercial (CC BY-NC 4.0) license, which permits others to distribute, remix, adapt, build upon this work noncommercially, and license their derivative works on different terms, provided the original work is properly cited and the use is non-commercial. See: http:// creativecommons.org/licenses/by-nc/4.0/

\section{REFERENCES}

1. Hardy MA, Acciai F, Reyes AM. How health conditions translate into self-ratings: a comparative study of older adults across Europe. $J$ Health Soc Behav 2014;55:320-41.

2. Eriksson I, Undén AL, Elofsson S. Self-rated health. Comparisons between three different measures. Results from a population study. Int J Epidemiol 2001;30:326-33.

3. Halford C, Welin C, Bogefeldt J, et al. A population-based study of nearly 15000 observations among Swedish women and men during 1973-2003. BMJ Open 2012;2:e001353.

4. Lorem GF, Schirmer H, Emaus N. Health impact index. Development and validation of a method for classifying comorbid disease measured against self-reported health. PLoS One 2016;11: e0148830.

5. Pèrez-Zepeda MU, Belanger E, Zunzunegui MV, et al. Assessing the validity of self-rated health with the short physical performance battery: a cross-sectional analysis of the international mobility in aging study. PLoS One 2016;11:e0153855.

6. Jylhä M. What is self-rated health and why does it predict mortality? Towards a unified conceptual model. Soc Sci Med 2009;69:307-16.

7. DeSalvo KB, Bloser N, Reynolds K, et al. Mortality prediction with a single general self-rated health question. A meta-analysis. J Gen Intern Med 2006;21:267-75.

8. Halford C, Ekselius L, Anderzen I, et al. Self-rated health, life-style, and psychoendocrine measures of stress in healthy adult women. Ups J Med Sci 2010;115:266-74.

9. Idler EL, Benyamini Y. Self-rated health and mortality: a review of twenty-seven community studies. J Health Soc Behav 1997;38:21-37.

10. Halford C, Anderzén I, Arnetz B. Endocrine measures of stress and self-rated health: a longitudinal study. J Psychosom Res 2003;55:317-20.

11. Haring R, Feng YS, Moock J, et al. Self-perceived quality of life predicts mortality risk better than a multi-biomarker panel, but the combination of both does best. BMC Med Res Methodol 2011;11:103.

12. Hackett ML, Yapa C, Parag V, et al. Frequency of depression after stroke: a systematic review of observational studies. Stroke 2005;36:1330-40.

13. Campbell Burton CA, Murray J, Holmes J, et al. Frequency of anxiety after stroke: a systematic review and meta-analysis of observational studies. Int J Stroke 2013;8:545-59. 
14. Tully $P$, Baune B. Comorbid anxiety disorders alter the association between cardiovascular diseases and depression: the German National Health Interview and Examination Survey. Soc Psychiatry Psychiatr Epidemiol 2014;49:683-91.

15. Kjaergaard M, Arfwedson Wang CE, Waterloo K, et al. A study of the psychometric properties of the Beck Depression Inventory-II, the Montgomery and Asberg Depression Rating Scale, and the Hospital Anxiety and Depression Scale in a sample from a healthy population. Scand J Psychol 2014;55:83-9.

16. Steptoe A, Deaton A, Stone AA. Subjective wellbeing, health, and ageing. Lancet 2015;385:640-8.

17. Vinkers $\mathrm{CH}$, Joëls $\mathrm{M}$, Milaneschi $\mathrm{Y}$, et al. Stress exposure across the life span cumulatively increases depression risk and is moderated by neuroticism. Depress Anxiety 2014;31:737-45.

18. Mavaddat $\mathrm{N}$, Valderas JM, van der Linde $\mathrm{R}$, et al. Association of self-rated health with multimorbidity, chronic disease and psychosocial factors in a large middle-aged and older cohort from general practice: a cross-sectional study. BMC Fam Pract 2014;15:185.

19. Jacobsen BK, Eggen AE, Mathiesen EB, et al. Cohort profile: the Tromso Study. Int J Epidemiol 2012;41:961-7.

20. Derogatis LR, Lipman RS, Rickels K, et al. The Hopkins Symptom Checklist (HSCL): a self-report symptom inventory. Behav Sci 1974;19:1-15.

21. Søgaard AJ, Bjelland I, Tell GS, et al. A comparison of the CONOR Mental Health Index to the HSCL-10 and HADS. Norsk Epidemiol 2003;13:279-84.
22. Hayes AF, Preacher KJ. Statistical mediation analysis with a multicategorical independent variable. Br J Math Stat Psychol 2014;67:451-70.

23. Hayes AF. Introduction to mediation, moderation, and conditional process analysis: methodology in the social sciences kindle edition. Guilford Press, 2003.

24. Hayes AJ. Model Templates for PROCESS for SPSS and SAS 2013. http://www.afhayes.com/ (accessed 02.06.2015).

25. Ihlebaek C, Brage S, Eriksen HR. Health complaints and sickness absence in Norway, 1996-2003. Occup Med 2007;57:43-9.

26. Clarke A, Warren L. Hopes, fears and expectations about the future: what do older people's stories tell us about active ageing? Ageing Soc 2007;27:465-88.

27. Mannsverk J, Wilsgaard T, Mathiesen EB, et al. Trends in modifiable risk factors are associated with declining incidence of hospitalized and nonhospitalized acute coronary heart disease in a population. Circulation 2016;133:74-81.

28. Grinde B. An approach to the prevention of anxiety-related disorders based on evolutionary medicine. Prev Med 2005;40:904-9.

29. Gilbert P. Evolution and depression: issues and implications. Psychol Med 2006;36:287-97.

30. Nettle D. Evolutionary origins of depression: a review and reformulation. J Affect Disord 2004;81:91-102.

31. Kvamme JM, Wilsgaard T, Florholmen J, et al. Body mass index and disease burden in elderly men and women: the Tromsø Study. Eur $J$ Epidemiol 2010;25:183-93. 